\title{
Modelo metodológico de implementación de lean manufacturing ${ }^{1}$
}

\author{
Mónica Patricia Sarria Yépez $z^{2}$ \\ Fundación Universitaria Católica Lumen Gentium \\ msarria@unicatolica.edu.co \\ Guillermo Alberto Fonseca Villamarín ${ }^{3}$ \\ Fundación Universitaria Católica Lumen Gentium \\ gfonseca@unicatolica.edu.co \\ Claudia Cristina Bocanegra-Herrera ${ }^{4}$ \\ Universidad Nacional de Colombia - Bogotá \\ cbocanegra@unal.edu.co
}

DOI: https://doi.org/10.21158/01208160.n83.2017.1825

Fecha de recepción: 17 de abril de 2017

Fecha de aprobación: 12 de junio de 2017

Cómo citar este artículo / To reference this article / Comment citer cet article / Para citar este artigo:

Sarria, M. P., Fonseca, G. A. y Bocanegra, C. C. (2017). Modelo metodológico de implementación de lean manufacturing. Revista EAN, 83, PP 51 - 71. https://doi.org/10.21158/01208160.n83.2017.1825

\section{Resumen}

Se diseñó una metodología flexible de implementación de lean manufacturing dirigido a empresas industriales, que partió de los modelos teóricos existentes. Se utilizó la metodología ICOM que permite determinar las relaciones entre los procesos y la construcción del diagrama de contexto de manera que la implementación de lean manufacturing sea más fácil de entender por las empresas. Se revisaron los principales métodos de implementación de diferentes autores que escriben sobre lean manufacturing identificando las 14 prácticas más usadas a través de una matriz comparativa, con lo cual se diseñó la metodología de implementación para empresas pequeñas y por personas con poca experiencia. En este sentido, el artículo se propone ofrecer una alternativa sencilla y ágil para el logro de una implementación exitosa de lean manufacturing, a través de los pasos que la metodología y el modelo proponen.

\section{Palabras clave}

Business process management, lean manufacturing, productividad, mejora continua.

${ }^{1}$ Producto derivado del proyecto de investigación Caracterización de lean production en Colombia, presentado por el grupo de investigación KIMSA de la Fundación Universitaria Católica Lumen Gentium.

${ }^{2}$ Ingeniera de producción de la Universidad Autónoma de Occidente, magíster en Logística Integral y Operaciones de la Universitat Oberta de Catalunya . Docente de la Fundación Universitaria Católica Lumen Gentium. ORCID: http://orcid. org/0000-0002-1439-3496

${ }^{3}$ Ingeniero de Producción de la Universidad Autónoma de Occidente, magíster en Logística Integral de la Universidad Autónoma de Occidente. Docente de la Fundación Universitaria Católica Lumen Gentium. ORCID: http://orcid.org/00000003-4379-2952

${ }^{4}$ Ingeniera Química de la Universidad del Valle, magíster en Ingeniería con énfasis en Ingeniería Industrial de la Universidad del Valle. Estudiante de doctorado en Ingeniería - Industria y Organizaciones de la Universidad Nacional de Colombia sede Bogotá. ORCID: http://orcid.org/0000-0002-8593-8879 


\section{Methodological model in the implementation of lean manufacturing}

Abstract. A flexible methodology for the implementation of lean manufacturing was design to be applied in the industrial company sector which started with existing theoretical models. The ICOM methodology was used to determine the relationship between processes and the construction of a context diagram making that the implementation of lean manufacturing be easily understood by companies. The main implementation methods from different authors who write about lean manufacturing were revised, identifying the fourteen most utilized practices using a comparative form, through which an implementation methodology was designed for small companies and unexperienced people. Consequently, this article proposes a simple and fast alternative to reach the successful implementation of lean manufacturing through the steps that this methodology and model suggest.

Key words. Business process management, lean manufacturing, productivity, continuing improvement.

\section{Modèle et méthodologie pour l'implémentation du lean manufacturings}

Résumé. Cet article présente la création d'une méthodologie flexible d'implémentation du lean manufacturing à partir de modèles théoriques existants et s'adressant aux entreprises industrielles. L'utilisation d'une méthodologie de type ICOM a permis de déterminer les relations entre les processus et la conception du diagramme pour que la mise en place du lean manufacturing soit plus simple à comprendre pour les entreprises. Les méthodes d'implémentation proposées par les principaux auteurs conceptualisant le lean manufacturing ont été passées en revue afin d'identifier, au travers d'une matrice comparative, les 14 pratiques les plus utilisées. Cette analyse préalable a ensuite permis de créer une méthodologie d'implémentation adaptée aux petites entreprises et aux personnes peu expérimentées. Cet article présente une alternative simple et efficace permettant une implémentation réussie du lean manufacturing grâce à la méthodologie et au modèle proposés.

Mots clefs. Processus de management des affaires, lean manufacturing, productivité, amélioration continue.

$$
\begin{gathered}
\text { Modelo metodológico de } \\
\text { implementação de lean manufacturing }
\end{gathered}
$$

Resumo. Desenhou-se uma metodologia flexível de implementação de lean manufacturing dirigida a empresas industriais, partindo dos modelos teóricos existentes. Utilizou-se a metodologia ICOM que permite determinar as relações entre os processos e a construção do diagrama de contexto, de maneira que a implementação de lean manufacturing seja mais fácil de entender por parte das empresas. Revisaram-se os principais métodos de implementação apresentados por diferentes autores que escrevem sobre lean manufacturing, identificando através de uma matriz comparativa as 14 práticas mais usadas, com o que se desenhou a metodologia de implementação para pequenas empresas e pessoa com pouca experiência. Neste sentido, o artigo visa oferecer uma alternativa simples e ágil para o alcance de uma implementação exitosa de lean manufacturing, através de etapas que a metodologia e o modelo propõem.

Palavras-chave. business process management, lean manufacturing, produtividade, melhora contínua. 


\section{Introducción}

Tean manufacturing es un conjunto de $L_{\text {principios y herramientas de gestión de }}$ la producción que busca la mejora continua a través de minimizar el desperdicio considerado este último como toda actividad que no agrega valor (Pérez Rave et al., 2011). La génesis de su desarrollo se puede ubicar, entonces, en el pionero y más emblemático caso de implementación que se observó en la Toyota Motor Corporation.

Lean manufacturing se ha convertido en una alternativa que ha mostrado su versatilidad al ser adoptada en los diferentes escenarios del sector industrial. En principio, esta filosofía comenzó a ser gestada luego de la devastación de la Segunda Guerra Mundial, donde países como Japón y Alemania sufrían los embates económicos de la posguerra. En la década de 1980, Toyota Motor Corporation venía trabajando en un modelo de sistema productivo que le permitiera mejorar su productividad, eficiencia y ser más competitiva (Ohno, 1991), lo cual se logró consolidar luego de que Taiichi Ohno asumiera como vicepresidente de esta compañia cuando se efectuó la implementación de su sistema de producción.

Este sistema sentó las bases para lo cual después sería el esquema de producción JIT (justo a tiempo). Por otro lado, en la década de 1990, se publicó el libro Machine that changed the world (Womack, Jones y Roos, 1990). En esta obra, se presentan las bases de la filosofía lean específicamente orientada a la producción, por ello, este concepto inicialmente desarrollado por Womack es fundamental para el desarrollo de proyectos bajo esta denominación.
En el contexto colombiano, pocas empresas realizan la implementación de lean de manera exitosa (Arrieta, Botero y Romano, 2010). Uno de los problemas fundamentales es la falta de metodologías de implementación práctica de la filosofía lean, lo cual también se evidencia en el mundo (Bakås, Govaert y Van Landeghem, 2011; Bhasin, 2012; Vienažindienè y Čiarnienè, 2013; Dora, Kumar, Van Goubergen, Molnar y Gellynck, 2012). En segundo término, los tiempos de implementación de lean manufacturing pueden ser de pocos meses o incluso de años (FortunySantos, Cuatrecasas Arbós, CuatrecasasCastellsaques y Olivella-Nadal, 2008), lo cual implicaría inversiones adicionales que impactan los presupuestos de estas empresas y por ello el nivel de implementación de lean manufacturing es reducido.

Es necesario fortalecer la relación entre universidades, Estado y empresas dado que esto incide en la configuración de esquemas productivos competitivos (Salazar y Valderrama, 2010; Howell, 2015), por eso, es importante que la academia estudie y ofrezca herramientas al sector productivo que vayan más allá del desarrollo de tesis, que es lo que usualmente se encuentra en las bases de datos universitarias y en países latinoamericanos.

El propósito de este artículo es presentar una metodología flexible para la implementación de lean manufacturing enfocada en empresas industriales en Colombia, partiendo de los modelos teóricos existentes, bajo unos criterios de selección de la secuencia de prácticas para una adecuada implementación de lean manufacturing, en el marco del proyecto Implementación de lean manufacturing 
en Colombia presentado a la Fundación Universitaria Católica Lumen Gentium.

Por otra parte, se establecen tres objetivos esenciales para la materialización de la implementación a través del modelo y la metodología.

- Caracterizar las prácticas y herramientas de lean manufacturing más utilizadas en un contexto empresarial, partiendo de la revisión documental que permita la implementación de modo sencillo y ágil.
- Desarrollar el modelo de implementación apoyado en la metodología ICOM-IDEF0, para el establecimiento de la estructura funcional, las etapas y las áreas que se inscriben dentro de una implementación de lean manufacturing en un contexto empresarial.

- Configurar la metodología para la implementación partiendo de los criterios propios del modelo.

\section{Método}

$\mathrm{P}$ ara la estructuración del modelo de implementación de lean manufacturing, se configuró una metodología que consiste en cuatro etapas fundamentales: i) revisión del estado actual con resultado de una matriz comparativa de las metodologías de implementación usadas por los escritores más representativos de la temática; ii) determinación de los criterios de selección del modelo; iii) selección del modelo; y iv) descripción del modelo.

A partir de la primera década del siglo XXI, la producción de literatura asociada a lean se ha enfocado en los esquemas productivos de industrias grandes con algunos elementos para PyME (Cabrera, 2012), y en algunos casos se deja a un lado la parte práctica que se requiere para los modelos de implementación de lean manufacturing,

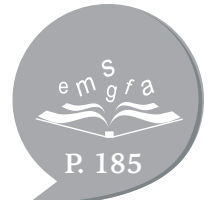
concentrando su atención en un enfoque más teórico (Modrák y Semančo, 2014).
Particularmente en Iberoamérica, los países que más han trabajado el aspecto de sistemas de producción asociados a lean han sido España, México, Brasil y Chile. En el caso de Colombia, esta temática es relativamente nueva, ya que en los últimos diez años ha venido cobrando importancia debido a que los esquemas productivos nacionales han estado orientados a la importación gracias a los periodos en los cuales la divisas mantuvieron unos precios asequibles, prueba de ello es el crecimiento industrial y competitividad reportado en el indicador doing business del Banco Mundial (2016).

Bajo este panorama, y según las perspectivas que marca el contexto global para los países en vías de desarrollo como Colombia, se hace necesario el trabajo para fortalecer el sector industrial mediante el uso de prácticas de lean, como lo presenta Pérez et al. (2011) en una empresa de confecciones de Colombia como una primera aproximación a la metodología de implementación de 
lean manufacturing, aunque no contempla la totalidad de sus herramientas y prácticas.

Dentro de los aspectos fundamentales de las prácticas lean, es importante destacar la estructura del sistema de producción según la disposición de los pilares lean que se determinaron en lo cual se conoce como la Casa Toyota, la que ha sido adaptada para una más amplia comprensión de las dimensiones que en ella se consideran (Figura 1).

Figura 1. Adaptación de la Casa Toyota

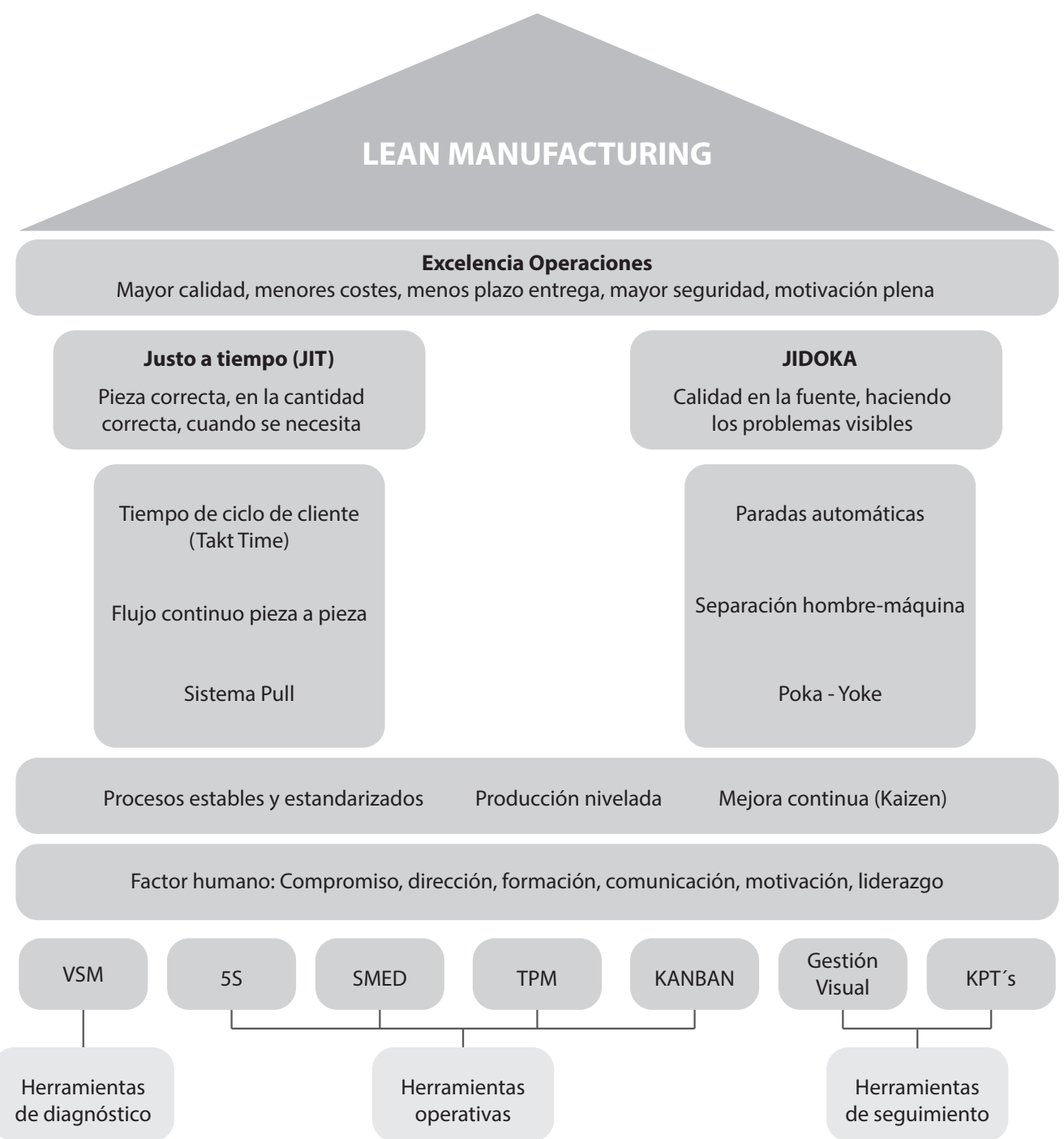

VSM: value stream map; SMED: single-minute exchange of die; TPM: total productive maintenance; KPI: key performance indicator.

Fuente. Hernández y Vizán, (2013, p. 18). 
De acuerdo con la información anterior, se muestra la base de la metodología lean, la cual está representada por las herramientas de diagnóstico, operativas y de seguimiento. En particular, como herramienta de diagnóstico se sugiere el mapa de flujo de valor o value stream map (VSM), donde se establecen los criterios para la construcción de un VSM actual (Singh y Sharma, 2009).

Dentro de las herramientas operativas se agrupan cuatro de las prácticas que presentan un mayor reconocimiento en el ámbito industrial. Se inicia con la herramienta $5 S$ que puntualiza en la adopción de prácticas asociadas a la disciplina, el orden y la limpieza previas a la ejecución de las labores y posteriores a ellas. En términos más coloquiales, significa ordenar la casa (Ohno, 1991). La siguiente práctica dentro de las herramientas operativas es la preparación rápida de máquinas o single-minute exchange of die (SMED) (Dillon y Shingo, 1985), que se centra en la reducción de tiempos de alistamiento entre los procesos y las máquinas que intervienen en el sistema productivo.

Por otra parte, se considera importante para la implementación de lean el uso de la práctica de mantenimiento productivo total o total productive maintenance (TPM), que se orienta a la adopción de métodos y acciones tendientes a mejorar los procesos asociados con los mantenimientos que se requieren en el sistema de producción de las empresas (Wireman, 2004). Finalmente, este conjunto de herramientas operativas se cierra con el uso de etiquetas de instrucción o Kanban (Sugimori, Kusunoki, Cho y Uchikawa, 1977).

Dentro de las herramientas de seguimiento propias de lean se determina la participación del control visual, que integrado al Kanban, permite la identificación de los componentes que circulan por la infraestructura productiva de la empresa y hace posible la adopción de técnicas para la detección de cuellos de botella, entre otras características. Como adición a lo anterior, está el uso de los indicadores clave de desempeño o key performance indicator (kpi), a través de los cuales es posible examinar el comportamiento de los procesos y los flujos del sistema productivo (Baudin, 2007).

Las anteriores herramientas no son aplicadas por las pequeñas empresas. Matt y Rauch (2013) afirman que estas últimas no aplican las prácticas lean manufacturing por iniciativa propia, a menos que esten integradas en una cadena de valor global; además presentan vacíos de la gestión gerencial y les es díficil contratar personal altamente calificado debido a las limitaciones de presupuesto. 


\section{Resultados}

Para estructurar el modelo propuesto de implementación de lean manufacturing, se tuvieron en consideración diferentes variables a la hora de lograr una metodología de implementación estandarizada, como la cultura regional, las prácticas empresariales, el tamaño de la empresa, la clasificación empresarial -manufactura o servicios-, entre otras. Por lo anterior, se hace complejo encontrar un modelo estándar de implementación - secuencias de prácticas entre los autores que estudian e implementan lean manufacturing; si se logra evidenciar es la falta de unificación de la cantidad de herramientas y prácticas que constituyen lean manufacturing. En este sentido, se encuentran autores que establecen alrededor de 100 técnicas disponibles (Pavnaskar, Gershenson y Jambekar, 2003), y otros autores como Espejo y Moyano (2007) que establecen 16; pero también se encuentran pocos referentes que presenten un modelo de implementación unificado que estandarice la secuencia de prácticas lean manufacturing. Terrubiano y Muñoz (2009) presentan un esquema de las técnicas y los conceptos más ampliamente difundidos por los principales estudiosos de lean manufacturing, que evidencia cómo a lo largo del tiempo los diferentes autores no han logrado unificar las prácticas lo cual no permite estandarizar un modelo de implementación.

Se observa que las prácticas más usadas y que perduran a lo largo del tiempo son 5S, mejoramiento continuo o Kaizen, Kanban, jalonamiento de la producción o pull y SMED, prácticas que son tenidas en cuenta en el modelo de implementación que se plantea en este artículo (Tabla 1).

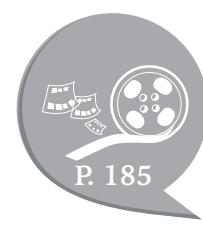

Tabla 1. Principales técnicas y conceptos ligados a lean manufacturing.

\begin{tabular}{|c|c|c|c|c|c|}
\hline \multicolumn{6}{|c|}{ Técnicas y conceptos ligados a lean manufacturing más ampliamente difundidos } \\
\hline $\begin{array}{l}\text { Técnicas y conceptos ligados a lean } \\
\text { manufacturing }\end{array}$ & $\begin{array}{c}\text { MONTWANI } \\
2003\end{array}$ & $\begin{array}{l}\text { SCHROER } \\
2004\end{array}$ & $\begin{array}{l}\text { BHAIN } \\
2006\end{array}$ & $\begin{array}{c}\text { WORLEY } \\
\& \text { DOOLEN } \\
2006\end{array}$ & $\begin{array}{l}\text { ABDULMALEK } \\
2007\end{array}$ \\
\hline $5 S$ & & SI & SI & SI & SI \\
\hline KAIZEN & & SI & SI & SI & \\
\hline KANBAM & & SI & SI & SI & SI \\
\hline PRODUCCIÓN PULL & & SI & SI & SI & \\
\hline SMED & & SI & SI & SI & SI \\
\hline MAPEO DE VALOR AÑADIDO & SI & SI & SI & SI & \\
\hline ELIMINACIÓN DE LAS SIETE MUDAS & & SI & SI & & \\
\hline TRABAJO ESTANDARIZADO & & SI & & & \\
\hline POKA YOKE & & SI & SI & & \\
\hline DISTRIBICACIÓN DE PLANTA & & SI & & & \\
\hline CALIDAD DE ORIGEN & & SI & & & \\
\hline
\end{tabular}


Tabla 1. Principales técnicas y conceptos ligados a lean manufacturing(Continuación).

\begin{tabular}{|l|c|c|c|c|c|}
\hline REDUCCIÓN DEL TAMAÑO DE LOTE & & SI & & & \\
\hline EQUIPO DE TRABAJO & SI & SI & & & \\
\hline $\begin{array}{l}\text { ALACENAMIENTO DE PUNTO DE } \\
\text { USO }\end{array}$ & SI & SI & & & \\
\hline FLUJO DE UNA PIEZA & SI & SI & SI & & \\
\hline CÉLULAS DE TRABAJO & SI & SI & SI & & SI \\
\hline TAKT TIME & SI & SI & & & SI \\
\hline JIT & & & & & SI \\
\hline TPM & & & SI & & SI \\
\hline TQM & & & & & \\
\hline EQUILIBRADO DE LÍNEAS & SI & & & & \\
\hline GESTIÓN DE CUELLOS DE BOTELLA & SI & & & & \\
\hline KAIKAKU & & & SI & & \\
\hline DESARROLLO DE PROVEEDORES & & & SI & & \\
\hline $\begin{array}{l}\text { REDUCCIÓN DEL NÚMERO DE } \\
\text { PROVEEDORES }\end{array}$ & & & SI & & \\
\hline
\end{tabular}

Fuente. Torrubiano y Machín, (2009).

\subsection{Comparativo de metodologías de implementación}

Existen diferentes metodologías de implementación que presentan diferencias entre ellas de acuerdo con el objetivo perseguido por sus autores, tamaño de la empresa objeto de aplicación y año de desarrollo (Womack y Jones, 2003; Kennedy y Maskel, 2006; Dennis, 2007; Rivera Cadavid, 2013; Black y Phillips, 2013). Aunque también comparten algunos elementos comunes e indispensables como el análisis de los procesos a través del VSM para luego diseñar e implementar planes de mejora usando las diferentes prácticas lean.

Se organizaron en orden cronológico los autores consultados que utilizaron cada una de las prácticas identificadas con los números del 1-14, y una columna con el número 15 que destaca cuando el autor realizó el desarrollo en empresas PyME (Tabla 2). En la última fila, se identifican las prácticas que conforman cada una de las etapas del modelo propuesto. Del análisis de la información disponible de los diferentes autores consultados se reflejan tres grandes bloques de uso: i) prácticas de mayor uso como SMED, TPM, Kaizen, 5S, formación del recurso humano y extensión de la aplicación de los conceptos a proveedores y clientes; ii) prácticas de uso medio, como implementación de sistema pull, takt time, Kanban y enfoque en PyMEs; iii) prácticas de menor uso, como: construcción de la visión por la alta gerencia, diagnóstico utilizando mapeo de la cadena de valor, organización de los productos por familias y poka-yoke. 
Los anteriores resultados, en especial las prácticas de mayor uso, coinciden con las prácticas lean manufacturing más usadas y que perduran a lo largo del tiempo, presentadas por Torrubiano y Muñoz (2009) anteriormente (Tabla 1), y que a su vez hacen parte de las herramientas operativas presentadas (Figura 1).

Con relación a los responsables de la ejecución de esta etapa, se debe partir inicialmente de la gerencia - representante legal, CEO [chief executive officer], entre otros-, y el personal que gestiona los sistemas productivos - jefes de producción, operaciones, logística, etc.- También es importante incluir las áreas de planeación, programación y control de las operaciones -jefes de planeación, compras, etc. -, que serán los líderes de implementación. A su vez, se deberá buscar la adhesión del personal táctico y operativo para que, a través de un proceso de selección, se definan los líderes de área - supervisores, operarios o colaboradores del nivel operativo-.

\subsection{Criterios de selección del modelo}

Dentro de los aspectos que se toman en consideración para la selección de las prácticas lean manufacturing analizadas $\mathrm{y}$ necesarias para estructurar el modelo de implementación, se determinó la siguiente configuración de criterios:

- Facilidad de acceso a la información.

- Nivel de adaptación al contexto PyME.

- Enfoque de mejora continua.

- Aportes para el sistema productivo.

- Flexibilidad de las actividades de implementación.
- Capacitación rápida y significativa del talento humano.

- Facilidad de modelación.

- Repetible en los diferentes sectores PyMEs industriales.

Los responsables de los criterios de selección para el modelo deben estar asociados directamente a los procesos y las operaciones que desde los aspectos funcionales tienen incidencia sobre las acciones de producción, manejo y control. Es muy importante que se incentive la participación del personal operativo.

\subsection{Selección del modelo de implementación}

El modelo propuesto se estructuró desde una metodología integration definition for function modeling (IDEFO), la cual permite determinar las relaciones entre los procesos y se orienta a construir el diagrama de contexto de la metodología business process management (BPM). Según ella, se materializa el modelo propuesto de cuatro etapas: i) iniciar, ii) preparar, iii) implementar $y$ iv) ajustar, en las cuales se encuentra una desagregación de tres niveles para cada una, donde se visualizan las catorce prácticas relacionadas más adelante (Tabla 2 ).

En esencia, el nuevo modelo propuesto parte de una primera etapa que busca el compromiso de la gerencia, selecciona líderes y equipos de implementación, y realiza el diagnóstico utilizando mapeo de la cadena de valor para presentar un mapa de la situación inicial y futura. Luego, pasa a una segunda etapa de preparación que incluye la formación e integración del recurso humano, construir indicadores centrados en la disminución de desperdicios e implementar $5 \mathrm{~S}$ como una de las prácticas más sencillas y fáciles de lograr 
que motivan al personal a no desfallecer en el proceso de implementación de lean manufacturing. En la tercera etapa, se organizan los productos por familias y se implementa el takt time bajo un sistema pull. Por último, la cuarta etapa incluye las prácticas más representativas de lean manufacturing como SMED, TPM, Kaizen, poka-yoke y se propende por la extensión de la aplicación de los conceptos lean manufacturing a proveedores y clientes.

En adelante (Figuras 2 a 5), se representa la desagregación de las cuatro etapas del modelo propuesto y se visualizan las 14 prácticas incluidas en la matriz comparativa (ver Tabla 2). En dicha matriz comparativa, de los 23 autores consultados en la revisión de la metodología de implementación de lean manufacturing, se encontró que los autores con mayor número de prácticas asociadas al modelo propuesto en este artículo son Hernández y Visán (2013), Cabrera (2012),
Womack y Jones (2003) y Chakravorty (2009). Para esta etapa, los responsables deben estar de acuerdo con cuáles de las prácticas y herramientas tienen aceptación dentro del contexto interno de la empresa. Para ello, se debe conformar un equipo multidisciplinario donde es clave que participe la gerencia general, la planeación y las áreas asociadas a la producción. Esta puede tomar un tiempo que se calcula dependiendo de las oportunidades de mejora detectadas por el equipo de líderes -líderes de implementación y líderes de áreas-. En términos aproximados, se habla de alrededor de seis meses a un año, dado que tiene que ver con la adopción de proyectos desde un contexto operativo. Ahora bien, la implementación de las mejoras puede tener un tiempo aproximado de ocho meses considerando los ajustes y cambios escalonados para consolidar el mejoramiento continuo.

Tabla 2. Matriz de comparación de metodologías de lean manufacturing

\begin{tabular}{|c|c|c|c|c|c|c|c|c|c|c|c|c|c|c|c|}
\hline $\begin{array}{l}\text { Prácticas más } \\
\text { usadas para } \\
\text { implementación } \\
\text { lean manufacturing }\end{array}$ & 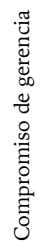 & 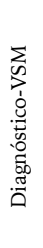 & 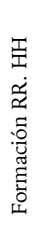 & $\curvearrowleft$ & 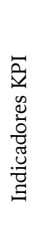 & 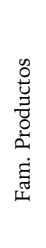 & $\bar{\Xi}$ & 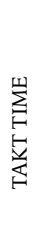 & $\begin{array}{l}\text { ज్ } \\
\text { స్ } \\
\text { ज్ }\end{array}$ & 蚖 & $\sum_{\hat{H}}$ & 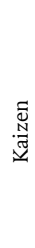 & 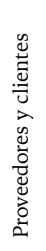 & 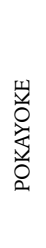 & $\sum_{i=1}^{w}$ \\
\hline Autores & 1 & 2 & 3 & 4 & 5 & 6 & 7 & 8 & 9 & 10 & 11 & 12 & 13 & 14 & 15 \\
\hline $\begin{array}{l}\text { Felizzola y Luna } \\
\text { (2014) }\end{array}$ & $\mathrm{x}$ & $\mathrm{x}$ & $\mathrm{x}$ & $\mathrm{x}$ & $\mathrm{x}$ & & & & $\mathrm{x}$ & & & $\mathrm{x}$ & $\mathrm{x}$ & $\mathrm{x}$ & $\mathrm{x}$ \\
\hline $\begin{array}{l}\text { Hernández y } \\
\text { Vizán (2013) }\end{array}$ & $\mathrm{x}$ & $\mathrm{x}$ & $\mathrm{x}$ & $\mathrm{x}$ & $\mathrm{x}$ & $\mathrm{x}$ & $\mathrm{x}$ & $\mathrm{x}$ & $\mathrm{x}$ & $\mathrm{x}$ & $\mathrm{x}$ & $\mathrm{x}$ & $\mathrm{x}$ & $\mathrm{x}$ & $\mathrm{x}$ \\
\hline Rivera (2013) & & $\mathrm{x}$ & $\mathrm{x}$ & $\mathrm{x}$ & $\mathrm{x}$ & & $\mathrm{x}$ & $\mathrm{x}$ & $\mathrm{x}$ & $\mathrm{x}$ & $\mathrm{x}$ & $\mathrm{x}$ & $\mathrm{x}$ & $\mathrm{x}$ & \\
\hline Cabrera (2012) & & $\mathrm{x}$ & $\mathrm{x}$ & $\mathrm{x}$ & $\mathrm{x}$ & $\mathrm{x}$ & $\mathrm{x}$ & $\mathrm{x}$ & $\mathrm{x}$ & $\mathrm{x}$ & $\mathrm{x}$ & $\mathrm{x}$ & $\mathrm{x}$ & $\mathrm{x}$ & $\mathrm{x}$ \\
\hline Dennis (2010) & $\mathrm{x}$ & & $\mathrm{x}$ & $\mathrm{x}$ & $\mathrm{x}$ & $\mathrm{x}$ & $\mathrm{x}$ & $\mathrm{x}$ & $\mathrm{x}$ & $\mathrm{x}$ & $\mathrm{x}$ & $\mathrm{x}$ & $\mathrm{x}$ & $\mathrm{x}$ & \\
\hline $\begin{array}{l}\text { Chakravorty } \\
\text { (2009) }\end{array}$ & $\mathrm{x}$ & $\mathrm{x}$ & $\mathrm{x}$ & $\mathrm{x}$ & $\mathrm{x}$ & $\mathrm{x}$ & $\mathrm{x}$ & & & $\mathrm{x}$ & $\mathrm{x}$ & $\mathrm{x}$ & $\mathrm{x}$ & $\mathrm{x}$ & $\mathrm{x}$ \\
\hline
\end{tabular}


Tabla 2. Matriz de comparación de metodologías de lean manufacturing(Continuación).

\begin{tabular}{|c|c|c|c|c|c|c|c|c|c|c|c|c|c|c|c|}
\hline $\begin{array}{l}\text { Fortuny-Santos, } \\
\text { Cuatrecasas, } \\
\text { Cuatrecasas- } \\
\text { Castellsaque y } \\
\text { Olivella-Nadal } \\
(2008)\end{array}$ & & $\mathrm{x}$ & $\mathrm{x}$ & $\mathrm{x}$ & $\mathrm{x}$ & & $\mathrm{x}$ & & & $\mathrm{x}$ & & & & & \\
\hline $\begin{array}{l}\text { Kennedy y Maskel } \\
\text { (2006) }\end{array}$ & $\mathrm{x}$ & & $\mathrm{x}$ & & $\mathrm{x}$ & $\mathrm{x}$ & & & & & & $\mathrm{x}$ & $\mathrm{x}$ & & \\
\hline $\begin{array}{l}\text { Real, Pralus, } \\
\text { Pillet y Guizzi } \\
(2007)\end{array}$ & & & & $\mathrm{x}$ & $\mathrm{x}$ & & & $\mathrm{x}$ & $\mathrm{x}$ & $\mathrm{x}$ & & & & & $\mathrm{x}$ \\
\hline $\begin{array}{l}\text { Antony y Kumar } \\
\text { (2011) }\end{array}$ & & & & & $\mathrm{x}$ & & & & $\mathrm{x}$ & $\mathrm{x}$ & $\mathrm{x}$ & $\mathrm{x}$ & & & $\mathrm{x}$ \\
\hline $\begin{array}{l}\text { Bhasin y Burcher } \\
\text { (2006) }\end{array}$ & & $\mathrm{x}$ & & $\mathrm{x}$ & $\mathrm{x}$ & & $\mathrm{x}$ & & & $\mathrm{x}$ & $\mathrm{x}$ & $\mathrm{x}$ & $\mathrm{x}$ & & \\
\hline Schroer (2004) & & $\mathrm{x}$ & $\mathrm{x}$ & $\mathrm{x}$ & & $\mathrm{x}$ & $\mathrm{x}$ & $\mathrm{x}$ & $\mathrm{x}$ & $\mathrm{x}$ & & $\mathrm{x}$ & & & \\
\hline $\begin{array}{l}\text { Thomas, Barton } \\
\text { y Chuke-Okafor } \\
\text { (2008) }\end{array}$ & & & $\mathrm{x}$ & & & & & & & & & $\mathrm{x}$ & & & $\mathrm{x}$ \\
\hline $\begin{array}{l}\text { Womack y Jones } \\
\text { (2003) }\end{array}$ & $\mathrm{x}$ & $\mathrm{x}$ & $\mathrm{x}$ & $\mathrm{x}$ & $\mathrm{x}$ & & $\mathrm{x}$ & $\mathrm{x}$ & $\mathrm{x}$ & $\mathrm{x}$ & $\mathrm{x}$ & $\mathrm{x}$ & $\mathrm{x}$ & $\mathrm{x}$ & \\
\hline $\begin{array}{l}\text { Amoako- } \\
\text { Gyampah y } \\
\text { Gargeya (2001) }\end{array}$ & & & $\mathrm{x}$ & & & & & & & $\mathrm{x}$ & & $\mathrm{x}$ & $\mathrm{x}$ & & $\mathrm{x}$ \\
\hline $\begin{array}{l}\text { Vaughn, Shields y } \\
\text { Gutowski (2002) }\end{array}$ & $\mathrm{x}$ & & $\mathrm{x}$ & $\mathrm{x}$ & $\mathrm{x}$ & $\mathrm{x}$ & & $\mathrm{x}$ & $\mathrm{x}$ & $\mathrm{x}$ & $\mathrm{x}$ & $\mathrm{x}$ & $\mathrm{x}$ & $\mathrm{x}$ & \\
\hline $\begin{array}{l}\text { Regan y Slattery } \\
(2000)\end{array}$ & $\mathrm{x}$ & $\mathrm{x}$ & $\mathrm{x}$ & $\mathrm{x}$ & & $\mathrm{x}$ & $\mathrm{x}$ & $\mathrm{x}$ & & $\mathrm{x}$ & $\mathrm{x}$ & $\mathrm{x}$ & $\mathrm{x}$ & & \\
\hline $\begin{array}{l}\text { Hines y Taylor } \\
(2000)\end{array}$ & $\mathrm{x}$ & $\mathrm{x}$ & $\mathrm{x}$ & $\mathrm{x}$ & $\mathrm{x}$ & $\mathrm{x}$ & $\mathrm{x}$ & & & $\mathrm{x}$ & $\mathrm{x}$ & $\mathrm{x}$ & $\mathrm{x}$ & $\mathrm{x}$ & \\
\hline $\begin{array}{l}\text { White, Pearson y } \\
\text { Wilson (1999) }\end{array}$ & & & & & $\mathrm{x}$ & & & $\mathrm{x}$ & $\mathrm{x}$ & & $\mathrm{x}$ & & & & $\mathrm{x}$ \\
\hline Lee (1993) & & & & $\mathrm{x}$ & & & & & & $\mathrm{x}$ & $\mathrm{x}$ & $\mathrm{x}$ & & & $\mathrm{x}$ \\
\hline $\begin{array}{l}\text { Gupta y Brennan } \\
\text { (1995) }\end{array}$ & & & $\mathrm{x}$ & & & & $\mathrm{x}$ & & & & & & $\mathrm{x}$ & & $\mathrm{x}$ \\
\hline $\begin{array}{l}\text { Inman y Mehra } \\
(1990)\end{array}$ & & & & & & & & $\mathrm{x}$ & & $\mathrm{x}$ & $\mathrm{x}$ & & & & $\mathrm{x}$ \\
\hline $\begin{array}{l}\text { Shingo y Dillon } \\
\text { (1989) }\end{array}$ & & & & & $\mathrm{x}$ & $\mathrm{x}$ & $\mathrm{x}$ & $\mathrm{x}$ & $\mathrm{x}$ & $\mathrm{x}$ & & & & $\mathrm{x}$ & \\
\hline $\begin{array}{l}\text { \# autores que } \\
\text { usan la práctica }\end{array}$ & 9 & 11 & 16 & 15 & 16 & 10 & 13 & 12 & 12 & 18 & 14 & 17 & 14 & 10 & 12 \\
\hline $\begin{array}{l}\text { Porcentaje } \\
\text { de uso de la } \\
\text { práctica }\end{array}$ & $39 \%$ & $48 \%$ & $70 \%$ & $65 \%$ & $70 \%$ & $43 \%$ & $57 \%$ & $52 \%$ & $52 \%$ & $78 \%$ & $61 \%$ & $74 \%$ & $61 \%$ & $43 \%$ & $52 \%$ \\
\hline $\begin{array}{l}\text { Etapas del } \\
\text { modelo } \\
\text { propuesto }\end{array}$ & \multicolumn{2}{|c|}{ E1: Iniciar } & \multicolumn{3}{|c|}{ E2: Preparación } & \multicolumn{4}{|c|}{ E3: Implementar } & \multicolumn{6}{|c|}{ E4 Ajustar } \\
\hline
\end{tabular}

1. Compromiso de gerencia; 2. Diagnóstico-VSM; 3. Formación RR. HH.; 4. 5S; 5. Indicadores KPI; 6. Organización de los productos en familia de productos; 7. Pull; 8. Takt time; 9. Kanban; 10. SMED; 11. TPM; 12. Kaizen; 13. Extensión de la aplicación de los conceptos lean manufacturing a proveedores y clientes; 14. Poka-yoke; 15. Enfoque en PyMEs.

Fuente. Elaboración propia. 


\subsection{Descripción del modelo propuesto}

La implementación de la filosofía lean manufacturing es una tarea compleja que va mucho más allá de las herramientas, por lo cual es importante el papel de la alta gerencia para el éxito de su implementación. Los responsables de esta etapa son los investigadores o los integrantes del equipo que la empresa seleccionó para conformar el equipo de implementación, dado que son los que se capacitan para liderar los procesos y la secuencia de actividades.

Figura 2. Diagrama de contexto ILM.

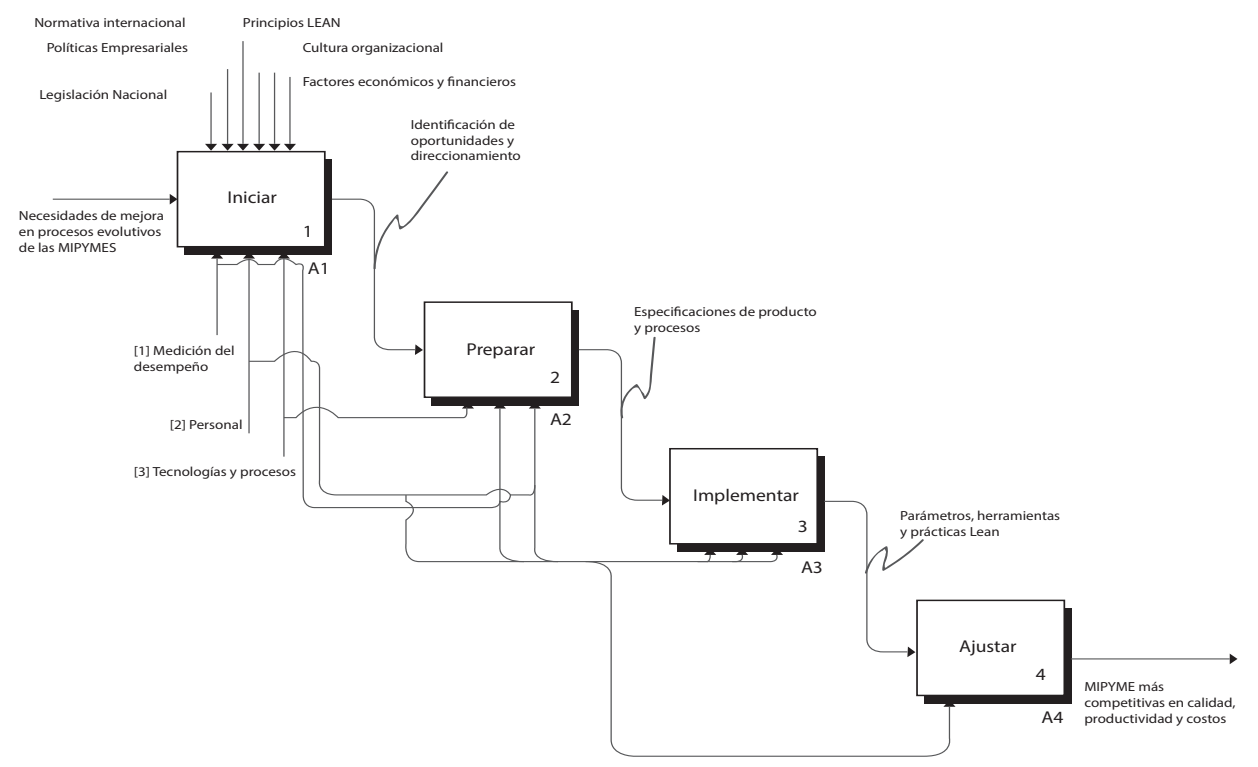

Fuente. Elaboración propia.

En la etapa iniciar (ver Figura 3), la alta gerencia decide comenzar la implementación de lean manufacturing en su empresa. Es importante que estén convencidos de realizar los cambios y que tenga el apoyo de los cargos más altos para así estructurar un plan motivacional, diseñado con las áreas de recursos humanos y la financiera de tal manera que consoliden premios - como bonos, dinero, oportunidad de ascenso, etc. - ; de no ser así, al presentarse las primeras dificultades es posible que desistan de continuar (Gómez, 2010). Para que esto no ocurra se debe construir una visión en la alta gerencia - práctica que es utilizada por el $39.1 \%$ de los autores consultados (ver Tabla 2) —, de tal manera que se den los espacios para lean manufacturing, así como liderar las reuniones de seguimiento y exigir los entregables pactados; luego encontrar líderes dentro de la organización que ayuden en la transmisión de esa visión al resto de los empleados.

Idealmente, los líderes deben estar acompañados de expertos (Scherrer-Rathje, Boyle y Deflorin (2009), pero manteniendo el liderazgo de manera que haya un aprendizaje efectivo, y así mismo, cuando el experto culmine su acompañamiento, todo el know-how 
permanezca dentro de la organización. Luego, comienza la subetapa de diagnóstico de la situación actual de la empresa haciendo énfasis en el área financiera de tal manera que justifique la implementación de lean manufacturing con la creación de indicadores que permitan medir el dinero ahorrado al reducir los desperdicios a través de este.

Para esta etapa de diagnóstico, también se usa la herramienta de VSM - práctica que es utilizada por el $47.8 \%$ de los autores consultados-. Esta técnica tiene varias ventajas: la primera corresponde a la realización de una manera sistémica y formal del mapeo que permite encontrar problemas ocultos; la segunda define las actividades que no agregan valor al producto; y la tercera permite la construcción de un mapa de estado futuro de los procesos una vez se hayan eliminado los problemas detectados inicialmente (Serrano, Ochoa y De Castro Vila, 2008). Esto se logra teniendo en cuenta que se debe llegar a un acuerdo - entre todo el equipo de implementación- de las metas por conseguir, para luego determinar las técnicas o los proyectos como camino para llegar al destino trazado con el VSM futuro.

Figura 3. Diagrama de etapa 1: iniciar.

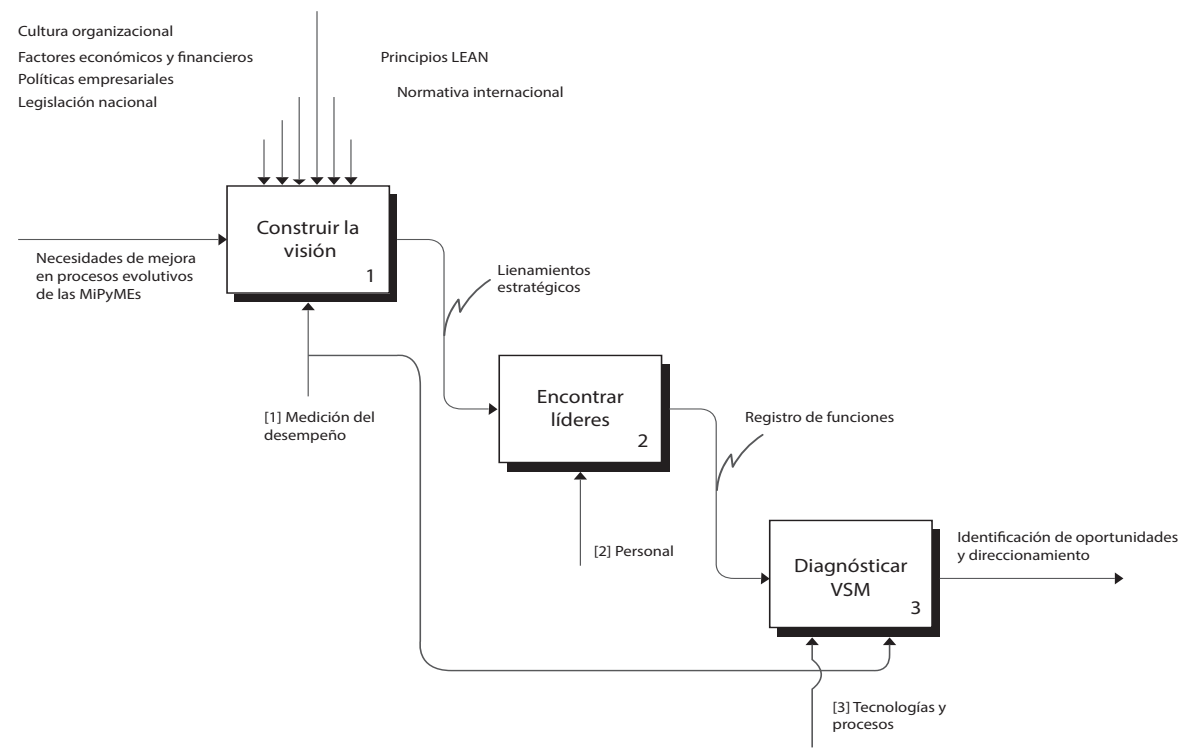

Fuente. Elaboración propia.

Culminada la etapa iniciar sigue la etapa de preparación (Figura 4). En este paso se organizan las condiciones y determinan los criterios bajo los cuales la empresa va a desarrollar la implementación. En primer lugar, se socializa y entrena a los empleados de mayor a menor cargo en la filosofía y principios lean, con el fin de disminuir la resistencia al cambio - práctica que es utilizada por el $70 \%-$ y como base fundamental para el éxito de la implementación. Esto se puede orientar como una academia donde los líderes son maestros de los demás empleados. Además, los empleados deben involucrarse en pequeños grupos de resolución de problemas (Bortolotti, Boscari y Danese, 2015).

Luego se implementan las 5S - práctica que es utilizada por el $65 \%$ de los autores consultados - en toda la organización, es 
decir, desde la bodega hasta las oficinas administrativas, lo cual permite ganar una cultura organizacional. Esta actividad se debe efectuar de tal manera que no se vean perjudicadas las labores cotidianas de los empleados. La configuración de esta parte del proceso es conceptualmente sencilla, pero requiere disciplina, por lo cual la cultura/idiosincrasia influye mucho en esta fase. Para implementar las 5S, primero, se debe clasificar - seiri- qué elementos son necesarios y cuáles no para luego deshacerse de estos últimos.

Después se organiza - seiton-, donde todos los elementos que se clasificaron como necesarios se deben ubicar e identificar de manera que sea fácil su utilización y la reposición sea rápida. Para esto, se ha de tener en cuenta que los objetos de uso frecuente deben estar más cerca. Además, para evitar deterioro por almacenamiento prolongado, hay que manejar un sistema first in, first out: primero en entrar, primero en salir (FIFO).

Una vez despejado y ordenado el espacio de trabajo, se debe mantener limpio - seiso-, porque la limpieza permanente en el sitio de trabajo contribuye a evitar el mal funcionamiento de la maquinaria y equipos, a la vez que facilita la ejecución de las actividades productivas; para esto, se debe intentar, como primera medida, no ensuciar.

Después de haber logrado los primeros tres pasos, se deben estandarizar - seiketsulos procesos de orden y limpieza cada día. Por lo cual la gestión visual es una parte crucial para este proceso ya que favorece la identificación de actividades que realizar por el personal de la empresa. Finalmente, se debe seguir mejorando - shitsuke-.

Figura 4. Diagrama de etapa 2: preparar.

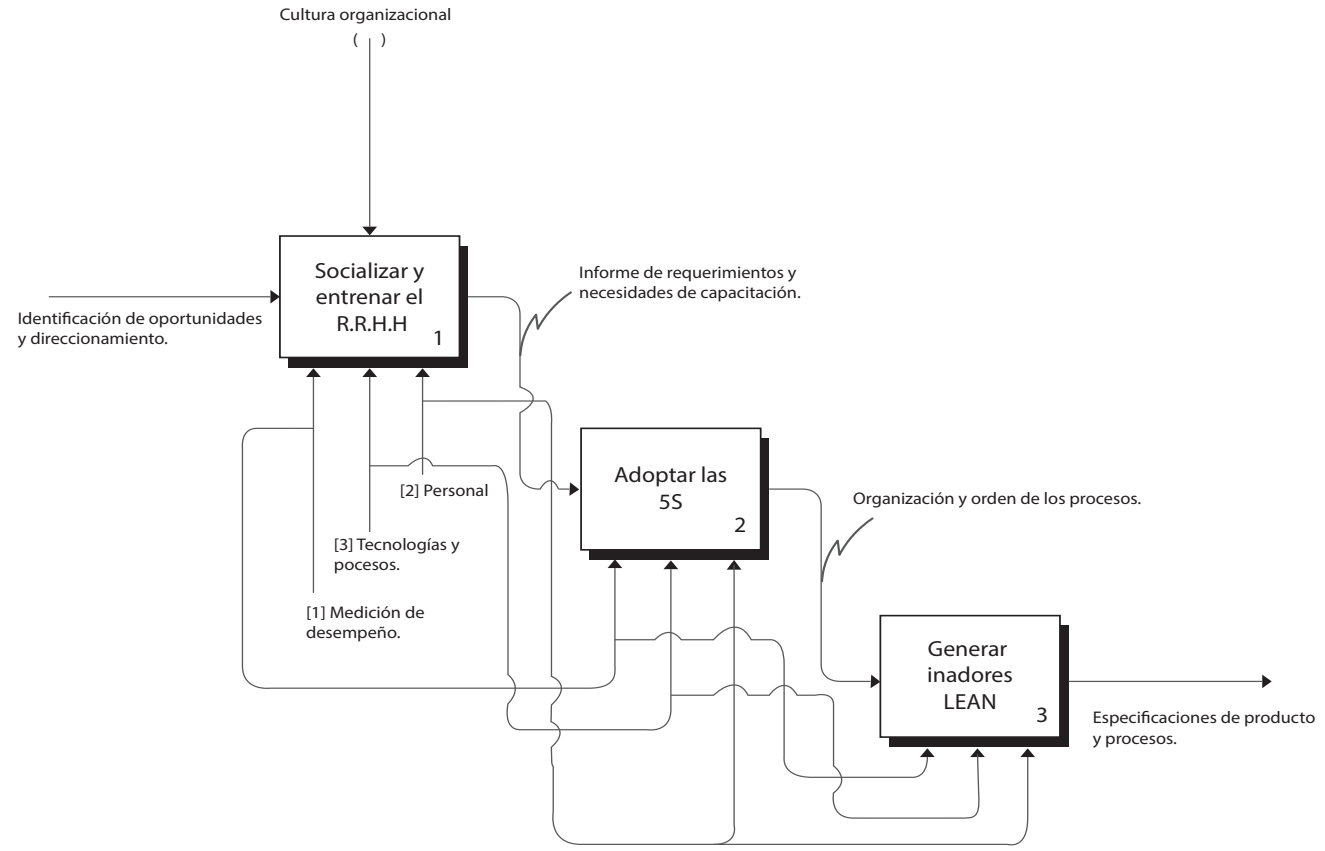

Fuente. Elaboración propia. 
Con la capacitación e implementación de 5S como práctica de lean manufacturing, se generan los indicadores y las metas por cumplir. Estos deben centrarse en la disminución de los desperdicios, con la participación del recurso humano, por lo cual se debe tener en consideración el talento y las habilidades de los empleados (De Haan, Naus y Overboom, 2012), así como las condiciones de ergonomía y seguridad en el trabajo. En las metas de reducción de los desperdicios debe haber un enlace con la disminución de inventario en proceso -WIP: work in progress - , los cuello de botella y los productos defectuosos (Villaseñor y Galindo, 2008).

En la tercera etapa implementación (ver Figura 5), se selecciona una familia de productos - práctica que es utilizada por el $43.5 \%$ de los autores consultados- o un producto estrella, que tenga un número importante de problemas asociados al inventario o a los defectos (Dennis, 2010).
Bajo esta consideración, es necesario establecer células de trabajo en la operación final, de manera que se adapte al ritmo de la demanda lo cual es conocido como takt time - práctica que es utilizada por el $52.2 \%$ de los autores consultados- (Black y Phillips, 2013). Las ventajas de las células de trabajo se orientan al mejor aprovechamiento del espacio dado que esto permite reducir el desplazamiento de los operarios y el movimiento de los materiales, además, una configuración flexible para el personal en el caso de requerirse un ajuste a la demanda. De manera similar se ejecuta para las otras operaciones aguas abajo y se conectan por medio de señales visuales Kanban - práctica que es utilizada por el $52.2 \%$ de los autores consultados-, es decir, manejando un sistema pull -empleado por el $56.5 \%$ de los autores consultados- (Rajadel y Sánchez, 2010). Esta etapa, después de ser afinada, se replica para las otras familias de productos.

Figura 5. Diagrama de etapa 3: implementación.

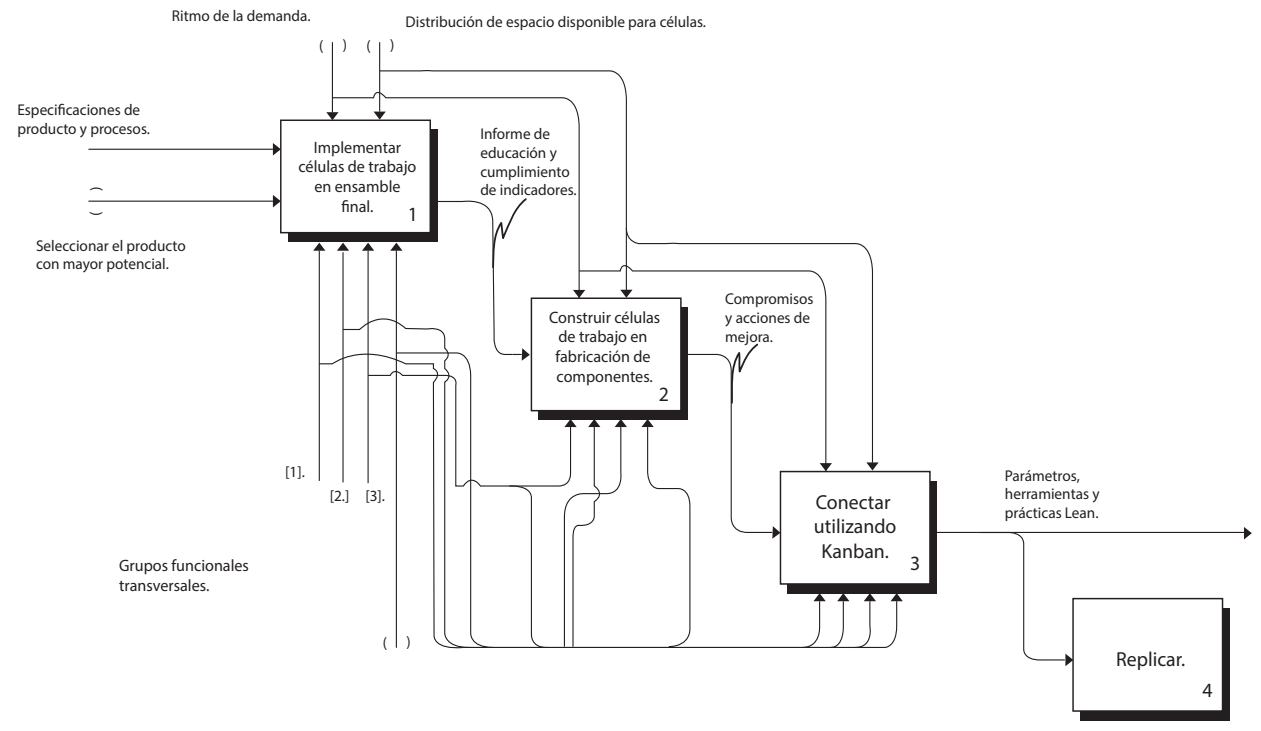

Fuente. Elaboración propia. 
Para la última etapa del modelo, ajustes (Figura 6), se requiere el uso del SingleMinute Exchange of Die (SMED) - práctica que es utilizada por el $78 \%$ de los autores consultados -, de manera que el flujo se suavice, se reduzca el tamaño del lote, lo cual evita la sobreproducción a la vez que contribuye a la reducción de las esperas de alistamiento de máquinas o equipos que son necesarios en los procesos de fabricación (Dillon y Shingo, 1985).

Figura 6. Diagrama de etapa 4: ajustes.

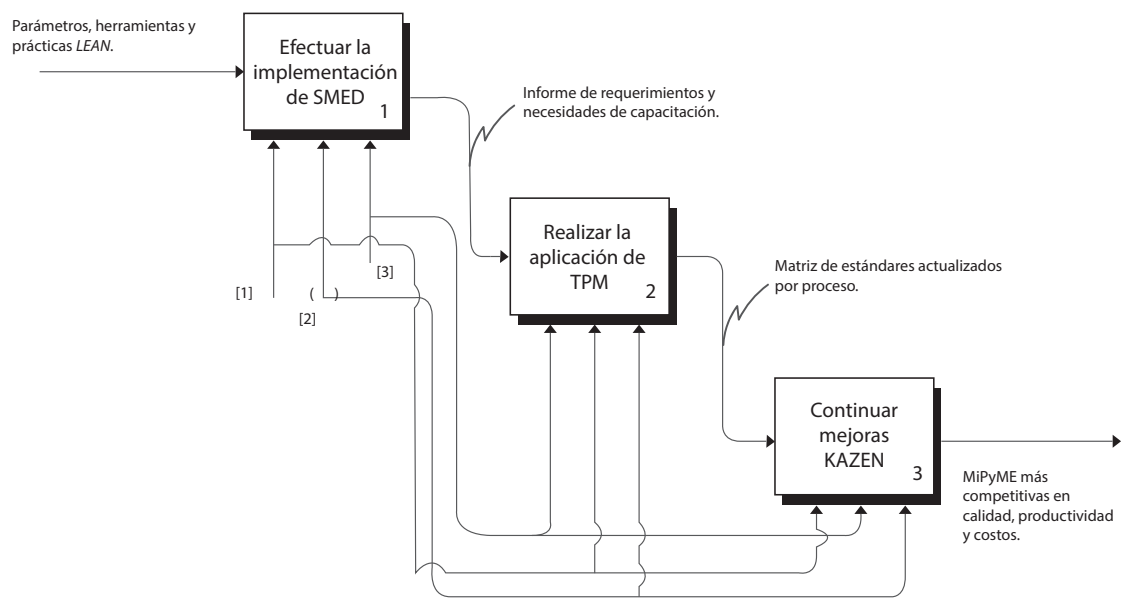

Fuente. Elaboración propia.

Como paso fundamental para la implementación de SMED, es clave que se efectúe la grabación de un video que contenga los métodos existentes de alistamiento y de esta manera se pueda hacer una lista y sus duraciones, en la cual se distingan entre los alistamientos internos y externos. Un alistamiento es interno solo si puede ser realizado cuando la máquina está apagada. Un alistamiento es externo si puede realizarse cuando la máquina está en funcionamiento, por tanto, trabajar para convertir los alistamientos internos en externos es vital para ganar tiempo, así como efectuar las actividades necesarias para eliminar o agilizar todos los alistamientos. Finalmente, documentar y fijar los nuevos procedimientos para que estos se traduzcan en un trabajo estandarizado.

El paso por seguir es implementar el total productive maintenance (TPM) - práctica que es utilizada por el $61 \%$ de los autores consultados - , para lo cual se debe identi- ficar cada pieza del equipamiento de las células; no es indispensable haber acabado la implementación completa, ya que es un proceso continuo. Luego, documentar el mantenimiento preventivo, orientado, en principio, a los equipos críticos y sus equipos de soporte, y finalmente para las demás piezas de equipamiento. Una vez cumplida la documentación, se desarrolla un cronograma de mantenimiento preventivo.

Es recomendable convertir los procedimientos en una lista de verificación fácil de usar y ubicarla cerca de las máquinas, así como desarrollar mediciones y gráficas para contrastar el cronograma versus las actividades de mantenimiento preventivo completadas. Es importante involucrar a los miembros del equipo en todos los mantenimientos preventivos y actividades asociadas a las reparaciones. 
Los principios y las técnicas lean deben ser compartimentados con los proveedores - práctica que es utilizada por el 61 $\%$ de los autores consultados-, en busca de manejar relaciones de confianza a largo plazo (Womack, Jones y Roos, 1990). El proceso de implementación de lean manufacturing nunca termina, ya que este se da en un ciclo de mejora continua conocido como Kaizen - práctica que es utilizada por el 74 $\%$ de los autores consultados-. Para esto, se puede utilizar el ciclo de Deming o plan, do, study and act (PDSA).. Este ciclo comprende cuatro pasos orientados a la generación de valor, aprendizaje y conocimiento. Todo lo anterior según la mejora continua de los procesos o del producto.

En el paso correspondiente a Plan, es donde se debe identificar la meta o formular el supuesto sobre el cual se trabajarán las métricas y se pondrán en marcha las acciones planificadas. El paso siguiente, Do hace relación a la manera como los componentes de la planificación son puestos en ejecución. El tercer paso, Study implica el análisis de los métodos y las prácticas asociadas con el sistema productivo para generar una estabilización de los procesos y el uso de las métricas. Por último, el paso cuatro, Act implica consolidar todos los elementos que garanticen la mejora y el cumplimiento de las metas o la verificación de los supuestos.

Lo anterior, bajo la óptica de lean manufacturing, significa continuar midiendo cada tiempo de alistamiento y las frecuencias de mantenimiento, lo cual consolida el control de las incidencias en cada operación. Además, el uso de un sistema de detección de errores basado en poka-yoke - práctica que es utilizada por el $43.5 \%$ de los autores consultados - permite el acoplamiento de las piezas de manera sencilla, también facilita el control visual, y disminuye los defectos al ensamblar componentes.

\section{Discusión}

T a metodología propuesta permitió Lidentificar las prácticas más usadas por los autores consultados de lean manufacturing, con lo cual se estructuró un modelo ajustado a las prácticas más efectivas para reducir las mudas, de tal manera que el flujo de producción sea más ágil.

El modelo propuesto de la metodología de implementación de lean manufacturing se desarrolló con el uso de las herramientas IDEF0, adaptadas al contexto industrial en Colombia, y es consecuente con los aspectos funcionales propios de las prácticas y herramientas de lean manufacturing las cuales se requieren para lograr que el paso a paso de la implementación y la integración visual y funcional de ellas - por medio de la gestión de procesos de negocio [BPM] se configure como un arreglo ordenado, lógico y que visibilice los componentes del modelo. De esta manera, se identifican cuáles son los elementos de entrada que alimentan el proceso, a su vez, se establecen los mecanismos de soporte para la gestión de dicho proceso y también se describen los controles o restricciones que tiene este. Así, finalmente, se indican cuáles son las salidas o los resultados del proceso actual. 
De acuerdo con lo anterior, hacer visibles las relaciones e interacciones del modelo genera un factor de cohesión con la metodología propuesta, dado que direcciona la secuencia de pasos desde la identificación de las características expuestas en los puntos

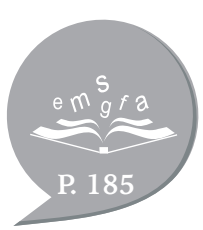
críticos asociados a la reducción de desperdicios, ajustes, estandarización y demás aspectos que inciden o impactan en el negocio. Bajo este marco de referencia, se establece la retroalimentación de cada proceso asociado con la implementación y el modelo, cada líder de área, sobre la base de Kaizen, puede incluir herramientas que van desde el control estadístico, indicadores y gráficas que permiten a los operarios conocer de primera mano la retroalimentación sobre las mejoras efectuadas. Por otra parte, el equipo de mejora deberá organizar unas reuniones periódicas para socializar los avances, las mejoras o los ajustes realizados.

\section{Conclusiones}

E 1 modelo desarrollado bajo la metodología de IDEFO y BPM permite visibilizar la secuencia de los pasos y la lógica que corresponde a las prácticas de lean manufacturing, su implementación y la relación con el sistema productivo - sustentado en los criterios de selección - . Así mismo, permite la visualización de la estructura funcional de las áreas que se inscriben en la implementación de lean manufacturing.

Como resultado del análisis del modelo propuesto, se concluye la importancia de realizar un detallado diagnóstico que permita identificar las causas que impactan negativamente en el desarrollo del sistema productivo, para así descubrir y eliminar los desperdicios a través de las diferentes prácticas lean. En este sentido, se desagregan las acciones que se deben efectuar para contribuir a la mejora, de manera que se incluya a los procesos que por su complejidad ofrecen mayores probabilidades de incidir en los niveles de desperdicios o mudas.
En el modelo propuesto, se requiere iniciar la implementación de lean manufacturing con la práctica 5S, ya que permite obtener un primer logro a corto plazo incentivando a la empresa a continuar con la implementación a pesar de los posibles obstáculos que se encuentren. Esta práctica, por sus beneficios con relación a la mejora en el orden y la configuración de las áreas de trabajo, motiva al personal a continuar fortaleciendo los hábitos productivos.

La propuesta realizada en este estudio reconoce la importancia de incluir la adopción de la disciplina como un factor necesario a trabajar con antelación, puesto que permite una rápida implementación de lean manufacturing.

Los desarrollos realizados en Colombia, apoyados con el uso de BPM para la implementación de lean manufacturing son limitados y exploran de manera tangencial las diferentes técnicas y prácticas de lean manufacturing. La secuencia que se propone en este artículo utilizando IDEF0 es un modelo el cual está en proceso de ser aplicado en una empresa del sector cuero. 


\section{Referencias}

Amoako-Gyampah, K. y Gargeya, V. B. (2001). Just-in-time manufacturing in Ghana. Industrial Management \& Data Systems, 101(3), 106-113. https://doi.org/10.1108/02635570110386562

Antony, J. y Kumar, M. (2011). Lean Six sigma: Research and practice. Bookboon.

Arrieta Posada, J. G., Botero Herrera, V. E. y Romano Martínez, M. J. (2010). Benchmarking sobre manufactura esbelta (lean manufacturing) en el sector de la confección en la ciudad de Medellín, Colombia. Journal of Economics, Finance and Administrative Science, 15(28), 141-170.

Bakås, O., Govaert, T. y Van Landeghem, H. (2011). Challenges and success factors for implementation of lean manufacturing in European SMES. En 13th International conference on the Modern Information Technology in the Innovation Processes of the Industrial Enterprise (MITIP 2011) (vol. 1). Trondheim, Norway: Tapir Academic Press. Recuperado de https://biblio.ugent.be/publication/1929995

Baudin, M. (2007). Working with machines: The nuts and bolts of lean operations with jidoka. Nueva York: Productivity.

Bhasin, S. (2012). Prominent obstacles to lean. International Journal of Productivity and Performance Management, 61(4), 403-425. https://doi. org/10.1108/17410401211212661

Bhasin, S. y Burcher, P. (2006). Lean viewed as a philosophy. Journal of Manufacturing Technology Management, 17(1), 56-72. https://doi. org/10.1108/17410380610639506

Black, J. T. y Phillips, D. T. (2013). lean engineering: The future has arrived. College Station, TX: Virtualbookworm.com Publishing Inc.

Bortolotti, T., Boscari, S. y Danese, P. (2015). Successful lean implementation: Organizational culture and soft lean practices. International Journal of Production Economics, 160, 182-201. https://doi. org/10.1016/j.ijpe.2014.10.013

Cabrera, R. (2012). Manual de lean manufacturing: simplificado para pymes. Editorial Academia Española.
Chakravorty, S. S. (2009). Six Sigma programs: An implementation model. International journal of production economics, 119(1), 1-16. Recuperado de http://www.sciencedirect.com/science/article/ pii/S0925527309000243

De Haan, J., Naus, F. y Overboom, M. (2012). Creative tension in a lean work environment: Implications for logistics firms and workers. International Journal of Production Economics, 137(1), 157-164.

Dennis, P. (2007). Getting the right things done: a leader's guide to planning and execution. Lean Enterprise Institute.

Dennis, P. (2010). Andy \& Me: Crisis \& Transformation on the lean Journey. Boca Raton, FL: CRC Press.

Dennis, P. (2015). Lean production simplified: A plainlanguage guide to the world's most powerful production system (3. ${ }^{\mathrm{a}}$ ed.). Boca Raton, FL: CRC press.

Dillon, A. P. y Shingo, S. (1985). A revolution in manufacturing: The SMED system. Portland, Oregon: Productivity Press.

Doing Business (2016). Doing Business 2016 midiendo la calidad y eficiencia regulatoria. Washington DC: International Bank for Reconstruction and Development/The World Bank.

Dora, M. K., Kumar, M., Van Goubergen, D., Molnar, A. y Gellynck, X. (2012). lean sigma implementation framework for food processing SMEs: the case of a Belgian confectionary. En 6th International conference on Decision Sciences for Performance Excellence. Hyderabad, India: IBS. Recuperado de https:// biblio.ugent.be/publication/3103818

Espejo Alarcón, M. y Moyano Fuentes, J. (2007). Lean production: estado actual y desafíos futuros de la investigación. Investigaciones Europeas de Dirección y Economía de la Empresa, 13(2), 179202. Recuperado de http://www.redalyc.org/ $\mathrm{html} / 2741 / 274120280010 /$

Felizzola Jiménez, H. y Luna Amaya, C. (2014). Lean Six Sigma en pequeñas y medianas empresas: un enfoque metodológico. Ingeniare: Revista Chilena de Ingeniería, 22(2), 263-277. https://doi. org/10.4067/S0718-33052014000200012 
Fortuny-Santos, J., Cuatrecasas Arbós, L., Cuatrecasas-Castellsaques, O. y Olivella-Nadal, J. (2008). Metodología de implantación de la gestión lean en plantas industriales. Universia Business Review, 20, 28-41. Recuperado de http://www. redalyc.org/html/433/43302003/

Gómez Botero, P. A. (2010). Lean manufacturing: flexibilidad, agilidad y productividad. Gestión \& Sociedad, 3(2), 75-88.

Gupta, S. M. y Brennan, L. (1995). Implementation of just-in-time methodology in a small company. Production Planning \& Control, 6(4), 358-364.

Hernández, J. C. y Vizán Idoipe, M. A. (2013). Lean manufacturing: concepto, técnicas e implantación. Escuela de Organización Industrial. Recuperado de https://www.eoi.es/savia/documento/eoi80094/lean-manufacturing-concepto-tecnicas-eimplantacion

Hines, P. y Taylor, D. (2000). Going lean: A guide to implementation. Cardiff: lean Enterprise Research Centre.

Howell, V. (8 abril 2015). Building Long-Term Success for Your Lean Initiative. En Ceramicindustry. Recuperado de http://www.ceramicindustry.com/ articles/94613-building-long-term-success-foryour-lean-initiative

Inman, R. A. y Mehra, S. (1990). The transferability of just-in-time concepts to American small businesses. Interfaces, 20(2), 30-37. https://doi. org/10.1287/inte.20.2.30

Kennedy, F. y Maskel, B. (2006). Accounting for the lean enterprise: Major changes to the accounting paradig. Intitute of Management Accountants Indiana University.

Lee, C. Y. (1993). The aplicabillity of just in time manufacturing to small manufacturing firms: An analysis. International Journal of Operations $\mathcal{E}$ Production Management, 13(4), 3-17.

Marmolejo, N., Milena Mejía, A., Pérez-Vergara, I. G., Rojas, J. A. y Caro, M. (2016). Mejoramiento mediante herramientas de la manufactura esbelta, en una Empresa de Confecciones. Ingeniería Industrial, 37(1), 24-35. Recuperado de http://scielo.sld.cu/scielo.php? pid =S181559362016000100004 \& script $=$ sci arttext\&tlng=en
Matt, D. T. y Rauch, E. (2013). Implementation of lean production in small sized enterprises. Procedia CIRP, 12, 420-425. https://doi.org/10.1016/j. procir.2013.09.072

Modrák, V. y Semančo, P. (2014). Handbook of research on design and management of lean production systems. Business Science Reference.

Ohno, T. (1991). El sistema de producción Toyota: más allá de la producción a gran escala. Barcelona: Gestión 2000.

Pavnaskar, S. J., Gershenson, J. K. y Jambekar, A. B. (2003). Classification scheme for lean manufacturing tools. International Journal of Production Research, 41(13), 3075-3090. https://doi. org/10.1080/0020754021000049817

Pérez Rave, J., La Rotta, D., Sánchez, K., Madera, Y., Restrepo, G., Rodríguez, M., ... y Parra, C. (2011). Identificación y caracterización de mudas de transporte, procesos, movimientos y tiempos de espera en nueve pymes manufactureras incorporando la perspectiva del nivel operativo. Ingeniare: Revista Chilena de Ingeniería, 19(3), 396-408. https://doi.org/10.4067/S071833052011000300009

Rajadel, M. y Sánchez, J. L. (2010). Lean manufacturing: la evidencia de una necesidad. Ediciones Díaz de Santos.

Ramírez Salazar, M. del P. y García Valderrama, M. (2010). La alianza universidad-empresa-Estado: una estrategia para promover innovación. Revista Escuela de Administración de Negocios, 68, 112-133.

Real, R., Pralus, M., Pillet, M. y Guizzi, L. (2007). A study of supporting programs for small and medium enterprises: A first stage going to "lean". En Industrial Engineering and Engineering Management, 2007 IEEE International Conference on (pp. 515-519). Singapur: IEEE. 10.1109/IEEM.2007.4419243

Regan, M. D. y Slattery, M. (2000). The Kaizen revolution: How to use Kaizen events to double your profits. Holden Press.

Rivera Cadavid, L. (2013). Justificación conceptual de un modelo de implementación de lean manufacturing. Heurística, 15, 91-106. 
Scherrer-Rathje, M., Boyle, T. A. y Deflorin, P. (2009). lean, take two! Reflections from the second attempt at lean implementation. Business Horizons, 52(1), 79-88. https://doi.org/10.1016/j. bushor.2008.08.004

Schroer, B. J. (2004). Simulation as a tool in understanding the concepts of lean manufacturing. Simulation, 80(3), 171-175. https://doi. org/10.1177/0037549704045049

Serrano Lasa, I., Ochoa Laburu, C. y de Castro Vila, R. (2008). An evaluation of the value stream mapping tool. Business Process Management Journal, 14(1), 3952. https://doi.org/10.1108/14637150810849391

Shingo, S. y Dillon, A. P. (1989). A study of the Toyota production system: From an Industrial Engineering Viewpoint. Nueva York: Productivity.

Singh, B. y Sharma, S. K. (2009). Value stream mapping as a versatile tool for lean implementation: an Indian case study of a manufacturing firm. Measuring Business Excellence, 13(3), 58-68. https:// doi.org/10.1108/13683040910984338

Sugimori, Y., Kusunoki, K., Cho, F. y Uchikawa, S. (1977). Toyota production system and kanban system materialization of just-in-time and respectfor-human system. The International Journal of Production Research, 15(6), 553-564. https://doi. org/10.1080/00207547708943149

Thomas, A., Barton, R. y Chuke-Okafor, C. (2008). Applying lean six sigma in a small engineering company:A modelforchange.JournalofManufacturing Technology Management, 20(1), 113-129. https:// doi.org/10.1108/17410380910925433

Torrubiano, J. y Muñoz Machín, I. (2009). Metodología LEAN en la Sanidad (Cuarta parte): construcción del pensamiento lean. Técnicas y herramientas. Forum Calidad, 21(207), 46-51.

Vaughn, M., Shields, T. y Gutowski, T. (2002). Summary of Research Conducted by the Manufacturing Systems Team 1994-2002. Massachusetts Institute of Technology. Recuperado de https://dspace.mit. edu/handle/1721.1/7520

Vienazindiene, M. y Ciarniene, R. (2013). lean manufacturing implementation and progress measurement. Economics and Management, 18(2), 366-373.
Villaseñor Contreras, A. y Galindo Cota, E. (2008). Conceptos y reglas de lean manufacturing (2. ${ }^{\mathrm{a}}$ ed.). México: Limusa.

White, R. E., Pearson, J. N. y Wilson, J. R. (1999). JIT Manufacturing: A Survey of Implementations in Small and Large U.S. Manufacturers. Management Science, 45(1), 1-15. https://doi.org/10.1287/ mnsc.45.1.1

Wireman, T. (2004). Total productive maintenance (2. ${ }^{\mathrm{a}}$ ed.). Nueva York. Industrial Press.

Womack, J. P. y Jones, D. T. (2003). lean thinking: banish waste and create wealth in your corporation (3. ${ }^{\mathrm{a}}$ ed.). Nueva York: Free Press.

Womack, J. P., Jones, D. T. y Roos, D. (1990). Machine that changed the world. Nueva York: Rawson Associates. 